\title{
Cardiac Metastasis from Medullary Thyroid Cancers with Long-Term Survival under Vandetanib
}

\author{
Camille Buffet $^{\mathrm{a}, \mathrm{b}}$ Sophie Leboulleux ${ }^{c}$ Françoise Kraeber-Bodéréd, e
}

Caroline Bodet-Milin d, e Laure Cabanes ${ }^{g, n} \quad$ Anthony Dohan $^{\text {h, }}{ }^{\mathrm{i}}$ Pascal Leprince ${ }^{\mathrm{j}}$

Martin Schlumberger ${ }^{\mathrm{b}, \mathrm{k}}$ Olivier Huillard ${ }^{\mathrm{f}, \mathrm{l}}$ Lionel Groussin $\mathrm{m}, \mathrm{n}, \mathrm{o}$

aDepartment of Thyroid Pathologies and Endocrine Tumors, AP-HP, Pitié-Salpêtrière Hospital, Groupe de Recherche Clinique ${ }^{\circ} 16$ Tumeurs Thyroïdiennes, Sorbonne Université, Paris, France; bUMR9019, Genome Integrity and Cancers, CNRS, Villejuif, France; 'Department of Nuclear Medicine and Endocrine Oncology, Gustave Roussy Institut, Villejuif, France; ${ }^{d}$ Nuclear Medicine Department, Université de Nantes, CHU de Nantes, CNRS, Inserm, CRCINA,

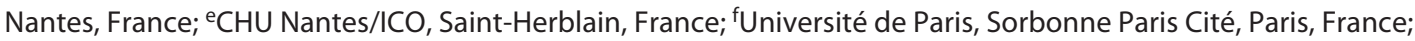
${ }^{9}$ Department of Cardiology, APHP, Cochin Hospital, Paris, France; hRadiology Department, Université de Paris, Paris, France; 'Department of Radiology, AP-HP, Hôpital Cochin, Paris, France; 'Department of Thoracic and Cardiovascular Surgery, Sorbonne Université, AP-HP, Pitié-Salpêtrière Hospital, Paris, France; ${ }^{k}$ Department of Nuclear Medicine and Endocrine Oncology, Gustave Roussy Institute, Villejuif, France; 'Department of Medical Oncology, AP-HP, Hôpital Cochin, Paris, France; mINSERM Unité 1016, CNRS UMR 8104, Institut Cochin, Paris, France; nUniversité de Paris, Paris, France; ${ }^{\circ}$ Department of Endocrinology, AP-HP, Hôpital Cochin, Paris, France

\section{Established Facts}

- Cardiac metastases from thyroid cancers are uncommon with a poor prognosis.

\section{Novel Insights}

- Our work highlights 2 major points:

- The diagnostic challenge represented by cardiac metastases: in both cases, the diagnosis was based on conventional imaging and originally confirmed by functional imaging with an immuno-PET scan using anti-CEA bispecific antibody and ${ }^{68} \mathrm{Ga}$-labeled peptide which is the ultimate evidence of the medullary thyroid origin.

- The long-term survival of 2 patients who have developed cardiac metastases from MTC, in the current era of personalized medicine with targeted therapy, has never been described in the previous literature.

\section{Keywords}

Medullary thyroid cancers $\cdot$ Cardiac metastasis $\cdot$ Rearranged during transfection inhibitors · Vandetanib - Rearranged during transfection mutation

\section{Abstract}

Background: Cardiac metastases from thyroid cancers are uncommon with a poor prognosis. There is a lack of longterm follow-up studies. Cases: We report 2 cases of cardiac
(C) 2021 The Author(s)

Published by S. Karger AG, Basel

This is an Open Access article licensed under the Creative Commons Attribution-NonCommercial-4.0 International License (CC BY-NC) (http://www.karger.com/Services/OpenAccessLicense), applicable to the online version of the article only. Usage and distribution for commercial purposes requires written permission.
Correspondence to:

Lionel Groussin, lionel.groussin@aphp.fr 
metastasis from medullary thyroid cancer (MTC). Both patients presented limited metastatic disease apart from a cardiac metastasis. The initial diagnosis was challenging and was facilitated by functional imaging with an immuno-PETCT using an anti-CEA bispecific antibody and a ${ }^{68} \mathrm{Ga}$-labeled peptide. Both patients were treated with the multitarget kinase inhibitor vandetanib with prolonged stability. The first patient was alive at the last follow-up, 14 years after the diagnosis of cardiac metastasis. The second patient required surgical excision of the cardiac mass because of disease progression under vandetanib. Conclusion: These cases illustrate long-term survival and effectiveness of clinical management of 2 patients who developed cardiac metastases from MTC, in the current era of personalized medicine with targeted therapy.

(C) 2021 The Author(s)

Published by S. Karger AG, Basel

\section{Introduction}

Cardiac metastases from medullary thyroid cancers (MTC) are uncommon. Their frequency in large autopsy series in patients with thyroid cancers is low, between 0 and $5 \%[1-5]$. Given the rarity of this metastatic location, the outcome remains unclear. The average length of survival is short, especially in patients with symptomatic and unresectable cardiac metastases [6]. This poor prognosis paradigm may be reconsidered in the light of current available therapies. We report here 2 cases of MTC cardiac metastasis with long-term outcome under the tyrosine kinase inhibitor vandetanib.

\section{Patient 1}

A 45-year-old woman was diagnosed with MTC after total thyroidectomy and neck dissection (Fig. 1a). Histology revealed an 11-mm thyroid tumor with minimal extra-thyroidal extension and massive lymph node metastases $\mathrm{pT} 1 \mathrm{~b}(\mathrm{~s}) \mathrm{N} 1 \mathrm{~b}$ (AJCC/TNM cancer staging system 8 th edition) [7]. Germline REarranged during Transfection $(R E T)$ gene analysis was negative. After a 3-year period of complete remission (calcitonin: $1 \mathrm{pg} / \mathrm{mL}$ ), a progressive rise in calcitonin (up to $945 \mathrm{pg} / \mathrm{mL}$, normal value $<10 \mathrm{pg}$ / $\mathrm{mL}$ ) and carcino-embryonic antigen (CEA) (up to $8.5 \mathrm{ng}$ / $\mathrm{mL}$, normal value $<5 \mathrm{ng} / \mathrm{mL}$ ) levels led to the diagnosis of costal metastases treated with external beam radiation and surgery, 8 years after initial diagnosis. Two years lat$\mathrm{er}$, a rise in calcitonin and CEA levels $(1,390 \mathrm{pg} / \mathrm{mL}$ and $25 \mathrm{ng} / \mathrm{mL}$, respectively) led to the diagnosis of a $21-\mathrm{mm}$ liver metastasis and a $30-\mathrm{mm}$ cardiac mass in the right ventricular apex. A DOPA (6-[18F]-L-fluoro-L-3, 4-dihydroxyphenylalanine) PET-CT and an immuno-PETCT using an anti-CEA bispecific antibody and a ${ }^{68} \mathrm{Ga}$ labeled peptide revealed cardiac uptake, leading to the diagnosis of MTC metastasis. Because of this threatening cardiac location and as an alternative to high-risk surgical treatment, radioimmunotherapy with iodine-131-labeled anti-CEA antibodies was performed. Two years after the cardiac metastasis diagnosis, the liver metastasis increased in size significantly and reached $26 \mathrm{~mm}$ with an increase in calcitonin and CEA levels $(19,662 \mathrm{pg} / \mathrm{mL}$ and $46 \mathrm{ng} / \mathrm{mL}$, respectively), and the patient was treated with vandetanib (300 mg/day), initially in a research protocol [8]. Notwithstanding the comparability between the different images performed, the cardiac metastasis remains globally stable after radioimmunotherapy. The liver metastasis, taken as a target lesion, decreased in size until complete response according to RECIST1.1 assessments, 7 years after the initiation of vandetanib. The cardiac metastasis decreased slightly on CT $(-4 \mathrm{~mm})$. No cardiological metastatic-related symptom was reported from the diagnosis. Vandetanib was responsible for moderate toxicity: asthenia, increase in the QTc interval (460-511 $\mathrm{ms}$ ), and photosensitization with the need for transitory withdrawal and/or reduced dosage. Finally, 10 years after its initiation, vandetanib was stopped because of severe functional renal failure. The evaluation at 2.5 years after vandetanib withdrawal showed a stable disease on imaging with stable tumoral marker levels (calcitonin at 272 $\mathrm{pg} / \mathrm{mL}$ and CEA at $31 \mathrm{ng} / \mathrm{mL}$ ), and the patient was still free of cardiological symptoms.

\section{Patient 2}

A 63-year-old woman was treated for a sporadic MTC with total thyroidectomy and neck dissection (Fig. 1b). Histology revealed a multifocal tumor with a foci of 20 $\mathrm{mm}$ in maximum diameter invading almost the entire lobe with minimal extra-thyroidal extension and massive lymph node metastases pT2(m)N1b [7]. After the initial surgery, serum calcitonin level dropped from 336 to 17 $\mathrm{pg} / \mathrm{mL}$. Three years later, calcitonin levels rose to up to $164 \mathrm{pg} / \mathrm{mL}$, leading to the diagnosis of lymph node metastases treated by 2 additional neck surgeries. Two years later, calcitonin and CEA levels increased $(751 \mathrm{pg} / \mathrm{mL}$ and $14.5 \mathrm{ng} / \mathrm{mL}$, respectively). A chest CT showed micronodular lung and liver metastases and a suspicious $65-\mathrm{mm}$ myocardial mass in the right atrioventricular groove in- 


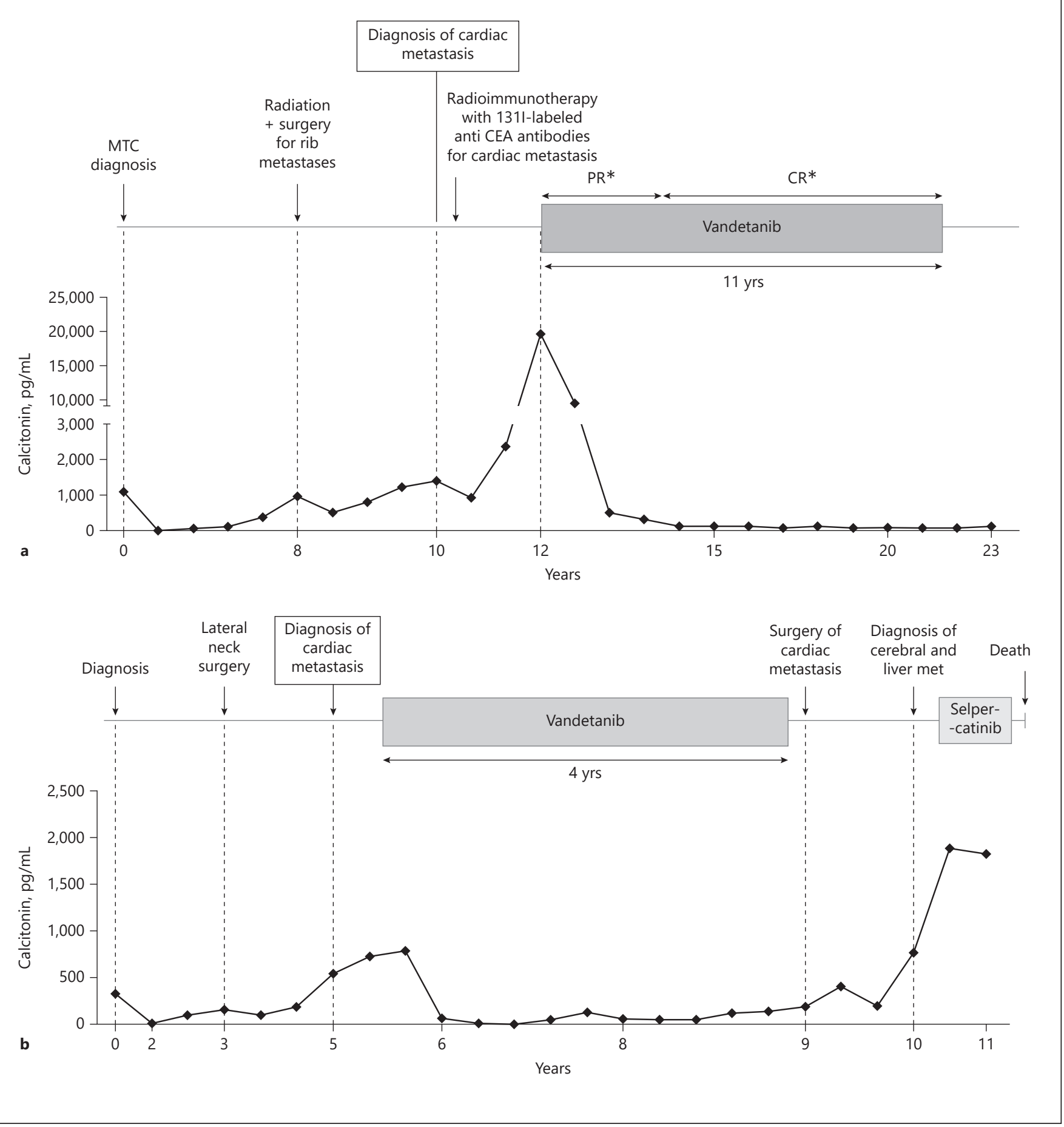

Fig. 1. Clinical and biochemical evolution of patient 1 (a). Clinical and biochemical evolution of patient 2 (b). MTC, medullary thyroid cancer; PR, partial response; CR, complete response. ${ }^{*}$ The target lesion was a liver metastasis. 
Fig. 2. a Cardiac CT in the coronal plane (left panel) and in the sagittal plane (right panel) showing the thyroid metastasis (red arrow) encasing the right ventricle and the right atrium with the right coronary artery (white arrow) encompassed by the tumor. b Planar image (left panel) and fused images (right panel) of an immuno-PET scan using an anti-CEA bispecific antibody and a ${ }^{68} \mathrm{Ga}$-labeled peptide revealing cardiac uptake of the metastatic lesion with a right lateral neck and mediastinal lymphadenopathies and a liver metastasis.
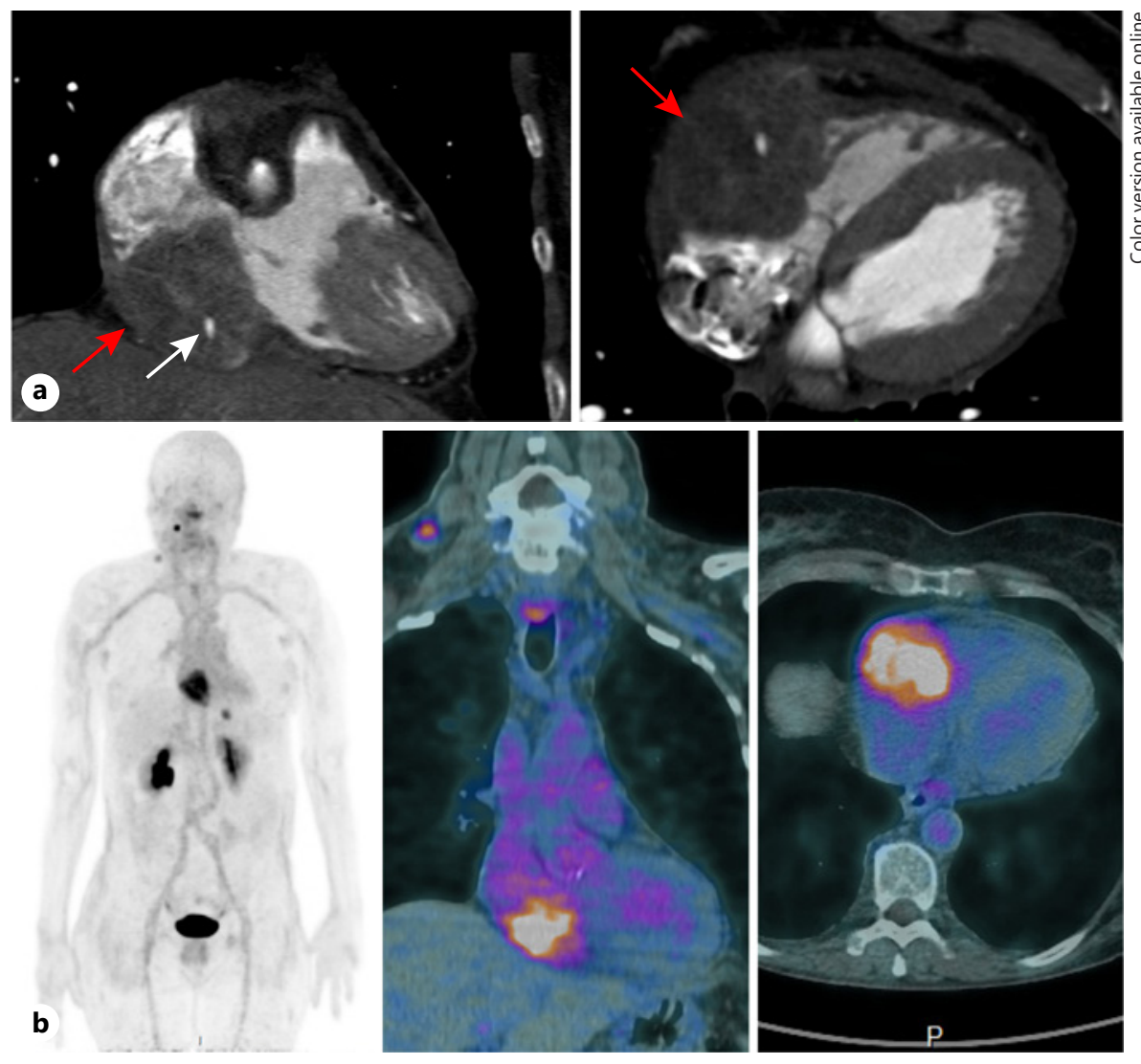

vading the pericardium, the ipsilateral atrial and ventricular cavities, and the tricuspid valve confirmed by a dedicated MRI (Fig. 2a). An F-DOPA PET-CT and ${ }^{111}$ In-octreoscan revealed a weak uptake of the cardiac mass. An immuno-PET-CT using an anti-CEA bispecific antibody and a ${ }^{68} \mathrm{Ga}$-labeled peptide (IMP288), performed within a clinical trial (NCT01730638), also revealed a cardiac uptake confirming the metastatic nature of the mass (Fig. 2b). At that time, there was no cardiac metastasisrelated consequence. The decision to perform a surgical resection was rejected given the unfavorable benefit-risk balance. Vandetanib (300 mg/day) was initiated, and a partial regression of the cardiac metastasis was observed $(-30 \%$ in 8 months) and a decrease in cervical lymph node and lung metastases. Vandetanib dosage reduction was necessary because of a prolonged QTc interval 9 months after its introduction. Progressively, calcitonin and CEA levels rose again $(422 \mathrm{ng} / \mathrm{mL}$ and $13.9 \mathrm{pg} / \mathrm{mL}$, respectively) in parallel with significant progression of the cardiac mass responsible for an atrioventricular obstacle and a low flow. Vandetanib was discontinued 3.5 years after its initiation, and wide surgical resection of the cardiac metastasis was performed with tricuspid biopros- thetic valve replacement. Histological analysis confirmed MTC metastasis with the proliferation marker Ki67 between 5 and $10 \%$. Given the partial metastasis resection, the progression of calcitonin to up to $199 \mathrm{pg} / \mathrm{mL}, 5$ months after the cardiac surgery, and the presence of a RET M918T somatic mutation, a treatment with cabozantinib was initiated (60 mg/day). However, due to a decrease in left ventricular function, cabozantinib was stopped 3 weeks later. As calcitonin and CEA levels decreased after these few weeks of treatment and because of the patient wish, the option of active surveillance was chosen. Unfortunately, 1 year later, new hepatic and brain metastases (right 25-mm frontal-parietal and left occipital lesions of few millimeters) were diagnosed in the workup of myoclonic seizures of the left hand. This diagnosis was accompanied by general progression of the metastatic disease (cardiac, pulmonary, and cervical lymph node metastases) along with calcitonin and CEA levels (respectively, $1,892 \mathrm{ng} / \mathrm{mL}$ and $146 \mathrm{pg} / \mathrm{mL}$ ). Stereotaxic radiation therapy by CyberKnife ${ }^{\circledR}$ was performed on brain metastases. The patient was included in a phase 1-2 trial evaluating the highly specific RET inhibitor selpercatinib [9]. Five weeks after selpercatinib initiation, the patient developed 
a confusional state. The MRI performed 8 days later revealed a worsening of a perilesional cerebral edema. The patient died 2 weeks later from brain herniation related to an increased vasogenic fronto-parietal edema with a mass effect on the right lateral ventricle.

\section{Discussion}

We report here 2 cases of unusual metastatic location of MTC. Giuffrida and Gharib [10] and Catford et al. [6] reviewed the literature of thyroid cancer cardiac metastasis. In the latest review, 59 cases were identified over a 130 -year period. The most common histological type is anaplastic thyroid cancer, closely followed by follicular thyroid cancer. Overall, the prognosis would appear poor [6]. Ten other cases of cardiac metastases have been reported since the last review [11-20], mostly from poorly differentiated or papillary carcinoma with aggressive features.

Only 3 cases of MTC cardiac metastasis have been reported [20-22]. One of them was our first patient reported in 2010 but without any outcome data [22]. The diagnosis of cardiac metastasis remains challenging. Ultrasonography, CT, or MRI is recommended in the first line [6]. MRI appears to be superior to ultrasound in the detection of cardiac metastases $[23,24]$. Some have suggested that echocardiography perfusion imaging could be used to provide information on the vascularization of the mass, helping to differentiate malignant from benign lesions [25]. Others have suggested that ${ }^{18} \mathrm{~F}-\mathrm{FDG}$ uptake on a PET-CT could be valuable to differentiate between benign, primary malignant, and metastatic cardiac tumors $[26,27]$. In the cases reported here, the endocrine nature of the cardiac mass was suggested by abnormal uptake on the F-DOPA PET scan and ${ }^{111}$ In-octreoscan. In both cases, iodine-131-labeled anti-CEA antibody uptake in the cardiac mass on an immuno-PET scan was the ultimate evidence of the medullary thyroid origin.

Patient-reported outcome in the literature is available for 1 MTC cardiac metastasis, with death 1 year after the diagnosis of the cardiac lesion [21]. The threatening metastatic location of our 2 patients motivated the introduction of a targeted therapy. The prolonged survival under vandetanib is a notable feature which has never been reported in MTC. Surgery initially recused in our second patient became finally mandatory despite the complexity of the procedure, as threatening cardiac-related symptoms occurred. The highly potent and selective RET inhibitors such as pralsetinib $[9,28]$ and selpercatinib [29] represent promising treatments in patients with driven RET-altered tumors and are currently tested, as first-line therapy, in worldwide phase 3 trials. The risk of cardiac rupture in case of rapid and important tumor regression remains an open question.

The conventional paradigm of short-term prognosis for patients who develop MTC cardiac metastases should be revised in the light of these new targeted therapies. Positive diagnosis remains challenging, but new functional imaging should allow recognizing the metastatic nature of a cardiac mass. In terms of clinical management, based on our experience, cardiac surgery is still indicated whenever technically feasible in case of cardiovascular impairment. However, when necessary, specific RET inhibitors should be considered based on their high efficacy and the lack of cardiovascular toxicity [9].

\section{Acknowledgment}

The authors acknowledge Liz Atzel for English language editing of the manuscript.

\section{Statement of Ethics}

Informed consent has been obtained from both patients for publication of the case report and accompanying images.

\section{Conflict of Interest Statement}

The authors have no conflicts of interest to declare.

\section{Funding Sources}

This research did not receive any specific grant.

\section{Author Contributions}

Contribution to the conception of the work: C.B., L.G., S.L., and M.S. Analysis or interpretation of data for the work: L.C., F.K.B., C.B.M., A.D., P.L., and O.H. Drafting the work: C.B., L.G., S.L., and M.S. Revising the work for important intellectual content: C.B., L.G., S.L., M.S., L.C., F.K.B., C.B.M., A.D., P.L., and O.H. Final approval of the version to be published: C.B., L.G., S.L., M.S., L.C., F.K.B., C.B.M., A.D., P.L., and O.H. Agreement to be accountable for all aspects of the work in ensuring that questions related to the accuracy or integrity of any part of the work are appropriately investigated and resolved: C.B., L.G., S.L., M.S., L.C., F.K.B., C.B.M., A.D., P.L., and O.H. 


\section{References}

1 Abraham KP, Reddy V, Gattuso P. Neoplasms metastatic to the heart: review of 3314 consecutive autopsies. Am J Cardiovasc Pathol. 1990;3(3):195-8.

2 Berge T, Sievers J. Myocardial metastases. A pathological and electrocardiographic study. Br Heart J. 1968;30(3):383-90.

3 Klatt EC, Heitz DR. Cardiac metastases. Cancer. 1990;65(6):1456-9.

4 Kline IK. Cardiac lymphatic involvement by metastatic tumor. Cancer. 1972;29(3):799808.

5 Pomorski L, Bartos M. Metastasis as the first sign of thyroid cancer. Neoplasma. 1999; 46(5):309-12.

6 Catford SR, Lee KT, Pace MD, Marasco SF, Longano A, Topliss DJ. Cardiac metastasis from thyroid carcinoma. Thyroid. 2011; 21(8):855-66.

7 Amin MB, Edge S, Greene F, Byrd DR, Brookland RK, Washington MK, et al. editor, AJCC cancer staging manual. 8th ed. New York, NY: Springer; 2017.

8 Wells SAJr, Robinson BG, Gagel RF, Dralle H, Fagin JA, Santoro M, et al. Vandetanib in patients with locally advanced or metastatic medullary thyroid cancer: a randomized, double-blind phase III trial. J Clin Oncol. 2012;30(2):134-41.

9 Wirth LJ, Sherman E, Robinson B, Solomon B, Kang H, Lorch J, et al. Efficacy of selpercatinib in RET-altered thyroid cancers. N Engl Med. 2020;383(9):825-35.

10 Giuffrida D, Gharib H. Cardiac metastasis from primary anaplastic thyroid carcinoma: report of three cases and a review of the literature. Endocr Relat Cancer. 2001;8(1):71-3.

11 Bertoldi EG, Severo MD, Scheffel RS, Foppa M, de Azevedo MJ, Maia AL. Left atrial metastases of poorly differentiated thyroid carcinoma diagnosed by echocardiography and magnetic resonance imaging-case report and review of literature. Echocardiography. 2012; 29(2):E30-3.

12 Giovanella L, Treglia G, Ceriani L, Weidner S, Perriard U, Bongiovanni M. Left atrial metastasis of Hürthle-cell thyroid carcinoma mimicking myxoma. J Nucl Cardiol. 2014;21(2): 406-7.
13 Ikeoka T, Ito T, Ando T. Left ventricular infiltration from thyroid papillary carcinoma mimicking the electrocardiographic changes of acute myocardial infarction. Endocrine. 2014;47(2):652-3.

14 Kaul S, Tulchinsky M, Campbell DB, Crist HS, Manni A. Isolated cardiac metastasis from papillary thyroid cancer: prolonged survival with late diagnosis related to inadequate positron emission tomography preparation. Thyroid. 2012;22(4):443-4.

15 Riva V, Bürgesser MV, Calafat P, Diller A, Ruades Ninfea JI, Caballero Escuti G. Metastatic cardiac tamponade as initial manifestation of papillary thyroid carcinoma. Medicina. 2011;71(6):550-2.

16 Rodriguez Caballero MG, Suarez Gutierrez L, Fernandez Fernandez L, Valdes Gallego N, Menendez Torre E. Cardiac tamponade as first sign of papillary thyroid carcinoma. Endocrinol Nutr. 2013;60(8):e1-2.

17 Tsoukalas N, Kostakis ID, Demiri S, Koumakis G, Barbounis V, Barbati K, et al. Neoplastic pericarditis as the initial manifestation of a papillary thyroid carcinoma. Ups J Med Sci. 2013;118(3):196-8.

18 Yanagisawa S, Suzuki Y, Yuasa T, Tanaka T. Right ventricular outflow tract obstruction: metastatic thyroid carcinoma. J Am Coll Cardiol. 2010;55(11):1159.

19 Yarmohammadi H, Tacher V, Faulhaber PF, Gilkeson RC, Aktay R, Kamouh A, et al. Imaging of dedifferentiated papillary thyroid carcinoma with left ventricular metastasis: a rare presentation of papillary thyroid metastatic disease. J Cancer Res Ther. 2013 JulSep;9(3): 490-2.

20 El Ghannudi S, Ben Abdelghani M, Germain $\mathrm{P}$, Blondet C, Romain B, Imperiale A. Cardiac metastases of small-bowel carcinoidadded value of (18)F-fluorodihydroxyphenylalanine positron emission tomography combined to magnetic resonance imaging. Circ Cardiovasc Imaging. 2019;12(2):e008405.
21 Bertagna F, Giubbini R, Savelli G, Pizzocaro C, Rodella C, Biasiotto G, et al. A patient with medullary thyroid carcinoma and right ventricular cardiac metastasis treated by $(90) \mathrm{Y}-$ Dotatoc. Hell J Nucl Med. 2009 MayAug; 12(2):161-4.

22 Morel O, Giraud P, Cahouet A, Lacoeuille F, Girault S. Visualization of cardiac metastasis from medullary thyroid carcinoma on F-18 DOPA PET/CT scan. Clin Nucl Med. 2010; 35(4):253-5.

23 Pavel M, Grossman A, Arnold R, Perren A, Kaltsas G, Steinmüller T, et al. ENETS consensus guidelines for the management of brain, cardiac and ovarian metastases from neuroendocrine tumors. Neuroendocrinology. 2010;91(4):326-32.

24 Pun SC, Plodkowski A, Matasar MJ, Lakhman Y, Halpenny DF, Gupta D, et al. Pattern and prognostic implications of cardiac metastases among patients with advanced systemic cancer assessed with cardiac magnetic resonance imaging. J Am Heart Assoc. 2016;5(5): e003368.

25 Moustafa SE, Sauvé C, Amyot R. Assessment of a right ventricular metastasis using contrast echocardiography perfusion imaging. Eur J Echocardiogr. 2008;9(2):326-8.

26 Meng J, Zhao H, Liu Y, Chen D, Hacker M, Wei Y, et al. Assessment of cardiac tumors by (18)F-FDG PET/CT imaging: histological correlation and clinical outcomes. J Nucl Cardiol. 2020.

27 Qin C, Shao F, Hu F, Song W, Song Y, Guo J, et al. 18F-FDG PET/CT in diagnostic and prognostic evaluation of patients with cardiac masses: a retrospective study. Eur J Nucl Med Mol Imaging. 2020;47(5):1083-93.

28 Subbiah V, Gainor JF, Rahal R, Brubaker JD, Kim JL, Maynard M, et al. Precision targeted therapy with BLU-667 for RET-driven cancers. Cancer Discov. 2018;8(7):836-49.

29 Subbiah V, Velcheti V, Tuch BB, Ebata K, Busaidy NL, Cabanillas ME, et al. Selective RET kinase inhibition for patients with RET-altered cancers. Annal Oncol. 2018;29(8):186976. 